\title{
Splenic Micronodular T-Cell/Histiocyte-Rich Large B-Cell Lymphoma: The Corticosteroid Pretreatment Hypothesis
}

\author{
Benzion Samuelia Karen Nalbandyan ${ }^{a} \quad$ Daniel Benharroch ${ }^{a} \quad$ Itai Levi $^{\mathrm{b}}$ \\ aDepartment of Pathology, Soroka University Medical Center, and Faculty of Health Sciences, Ben Gurion University \\ of the Negev, Beer-Sheva, Israel; ${ }^{b}$ Division of Hematology, Soroka University Medical Center, and Faculty of Health \\ Sciences, Ben Gurion University of the Negev, Beer-Sheva, Israel
}

\section{Keywords}

Splenic micronodular B-cell lymphoma · Corticosteroids ·

Apoptosis · Shrinkage

\section{Abstract}

Splenic micronodular T-cell/histiocyte-rich large B-cell lymphoma (THRLBCL) is derived from diffuse large B-cell lymphoma N.O.S., perhaps with some affinity with nodal THRLB$\mathrm{CL}$. Of note, in contrast with the latter, the only lymph nodes involved in association with the splenic micronodular pattern of the disease are the splenic hilar lymph nodes. The possibility that corticosteroids, when prescribed prior to splenectomy, cause histopathological and functional modulations, apoptosis, necrosis, tissue shrinkage, which may obscure the diagnostic morphological features of this variant lymphoma and cause an underdiagnosis of this condition. The indications for glucocorticoid therapy are either related to the lymphoma itself, or else to other comorbidities, like asthma and autoimmune disorders. We propose that patients with the splenic subset of the disease are likely to have been prescribed corticosteroids prior to histopathologic examination of the involved spleen, causing disparate morphologies. However, a reviewer might accidentally dismiss the corticosteroid pretreatment which is thus overlooked. Apoptosis, induced by corticosteroids, is hypothesized as the major mechanism initiating the histopathological and functional changes in the splenic micronodular variant of the lymphoma.

(C) 2021 The Author(s)

Published by S. Karger AG, Basel

\section{Introduction}

Splenic micronodular T-cell/histiocyte-rich large Bcell lymphoma (MTLBL) is a very rarely diagnosed entity, even considered at times of controversial nature [1]. This variant is probably derived from diffuse large B-cell lymphoma (DLBCL) and has some resemblance with T-cell/ histiocyte-rich large B-cell lymphoma (THRLBCL), to such a degree that the 2 might be confounded. The MTLBL variant of lymphoma occurs with splenomegaly, Bsymptoms, hypersplenism, and variable cytopenia [1]. Morphologically, it is composed of pale, disparate micronodules localized predominantly in the white pulp. Containing small-sized T-cells and histiocytes in the background, the micronodules encompass a limited number of large neoplastic B-cells, mainly centroblasts or

C 2021 The Author(s).

Published by S. Karger AG, Basel

This is an Open Access article licensed under the Creative Common Attribution-NonCommercial-4.0 International License (CC BY-NC) (http://www.karger.com/Services/OpenAccessLicense), applicable to the online version of the article only. Usage and distribution for commercial purposes requires written permission.
Correspondence to:

Daniel Benharroch, danielbenharroch1@gmail.com 
Reed-Sternberg-like cells. The histopathological features are probably analogous with those displayed by the spleen involved by THRLBCL and reaching stages III-IV [2]. In addition to pan-B markers (CD20; CD79a; CD19), these cells express BCL-6, some level of CD30, EMA and BCL2, but not CD23, CD10, or EBER [1, 3, 4].

Since some MTLBLs affect the bone marrow [3], and less frequently liver or lungs [3], this variant lymphoma should not be considered as a primary B-cell splenic lymphoma [5]. The differential diagnosis of this lymphoma subset comprises mainly Hodgkin's lymphoma, mainly nodular lymphocyte predominant Hodgkin lymphoma (NLPHL) [6]; DLBCL-NOS, THRLBCL, peripheral Tcell lymphoma, follicular lymphoma, and inflammatory granulomas.

In the absence of extensive clinical studies, and, depending on its being a variant of DLBCL, the limited number of patients reported so far with this lymphoma entity have been empirically treated with R-CHOP (rituximab, cyclophosphamide, doxorubicin, vincristine, and prednisone), with a predominantly poor outcome [1]. As the number of total splenectomies is markedly diminishing, the diagnosis of MTLBL may disappear entirely for practical purposes.

In the present study, an attempt is made to uncover the origin of the rare MTLBL variant. If indeed an association exists between MTLBL and THRLBCL, what is the trigger for the change? A thesis is advanced by which MTLBL may evolve following treatment with corticosteroids, prior to the diagnostic/therapeutic splenectomy.

\section{Materials and Methods}

The narrative review is set up primarily on 3 articles on MTLBL published by our group between 2008 and 2011 [6-8], since the results and conclusions thereof had not been interpreted to our complete satisfaction. Differences accounted for between MTLBL and THRLBCL had not been scrutinized in an exhaustive manner [1]. The apparent changes in the clinical course in patients with corticosteroid premedication might carry a therapeutic significance, distinct from the role of prednisone in the $\mathrm{R}-\mathrm{CHOP}$ protocol. The features of the Beer-Sheva (Israel) MTLBL cases will be summarized, together with a single case with pre-diagnosis treatment with corticosteroids and reported from the Emory University Hospital [3] and will be compared essentially with the cohort reported by Dogan et al. [1]. Moreover, the consequences elicited in these studies by the steroid pretreatment, have not been translated into practical clinical resolutions. We are determined, therefore, to bring to an end the inquiry on this rare variant of DLBCL. The present investigation will be established on the relevance of a pre-splenectomy corticoid therapy, on the splenic mass, on its morphology, on the clinical out- comes when available, and on a given propensity to miss the prior steroid treatment. Clinical features of patients with MTLBL will be compared with those of patients with DLBCL-NOS, THRLBCL, and NLPHL, considering the propensity for splenomegaly in all 4 entities.

\section{Results}

\section{Unfolding the Story}

In 2008, Kan et al. [7] reported on a 63-year-old woman who complained of fatigue, fever, and weight loss. Blood exams revealed anemia, hypo- $\gamma$-globulinemia and elevated LDH. Splenomegaly was noted, but no peripheral lymphadenopathy was identified on physical examination or by computed tomography scan. A bone marrow biopsy showed para-trabecular infiltrates, including of large B cells. On splenectomy, the spleen weighed $540 \mathrm{~g}$. Grossly, the white pulp was expanded, and the red pulp was preserved. Upon histology, the white pulp was invaded by micronodular infiltrates (Fig. 1a). Scattered CD20+ large tumor B cells, where found in a background of T-cells, histiocytes and eosinophils. A diagnosis of MTLBL was established. The patient was given 6 courses of R-CHOP. Complete remission was obtained and lasted 12 months, so far as the follow-up obtained. At the time of that report, Kan et al. [7] were not aware of a particular significance of the patient's clinical and laboratory findings: hypersplenism, fever, and splenomegaly. They were not mindful of the special context of the pre-splenectomy corticosteroid treatment, administered for symptomatic relief.

\section{The Second Episode}

In an attempt, to explore the effects of corticosteroids on lymphoid cells and hematolymphoid neoplasms, Kan et al. [6] identified 31 individuals who had been administered steroids prior to a diagnostic intervention. In addition to their inclusion in hematological chemotherapeutic protocols, glucocorticoids were prescribed for inflammatory and autoimmune conditions.

While the above study was in progress, 3 additional patients from our medical center were identified who were diagnosed as suffering from MTLBL and had received steroid treatment prior to splenectomy. One of them was S.M., the very patient described previously [7], but without being identified at then as receiving corticosteroids. Thus, despite a compelling clinical context, the steroid pretreatment prescribed was overlooked, until revision $[6,8]$. It is appreciated that this suggests that a sim- 

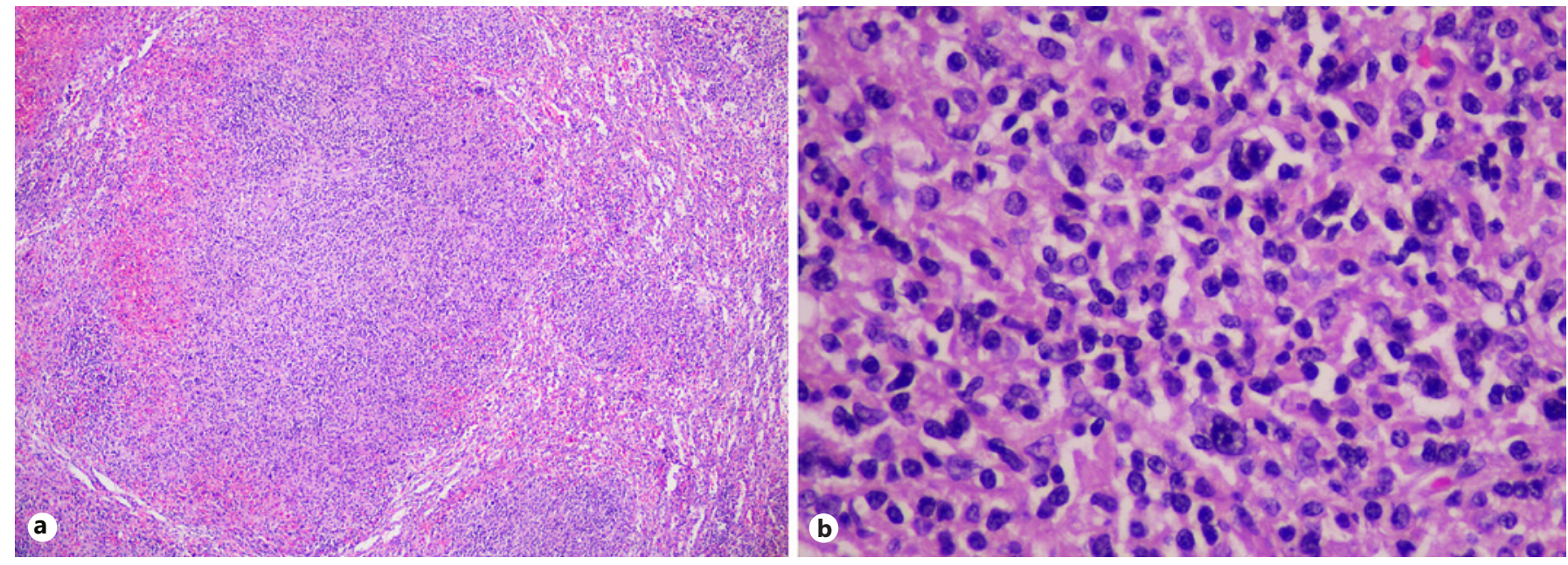

Fig. 1. a Section of the spleen of L.Y. A micronodule is found in the white pulp, surrounded by generally preserved red pulp $(\mathrm{H} \& \mathrm{E} . \times 40)$. b A close-up view of the micronodule in (a), displaying a lymphoid infiltrate, composed mainly of small lymphocytes, histiocytes and sparse large lymphoid cells (H\&E. ×400).

ilar negligence might recur, given the background conditions. A further MTLBL patient with prior corticoid therapy was described by Li et al. [3] as noted previously. In this case, the spleen weighed $780 \mathrm{~g}$.

Dogan et al. [1] did not report on corticosteroid premedication among their 17 cases with MTLBL. This contrasts highly with the 3 cases described from Israel [6-8]. Splenomegaly in the Beer-Sheva group varied from 540 to $850 \mathrm{~g}$, while in Dogan's cohort the range was 800 $2,500 \mathrm{~g}$. It is postulated that, in the Israeli group, the reduction in the splenic mass may be attributed to steroid induced shrinkage of the splenic parenchyma [6]. Our hypothesis on a probable role of the corticosteroids, when administered before splenectomy, underlines their effect on the splenic size, its morphologic variance, as well as regarding the infrequent occurrence of MTLBL and its outcome.

Considering the histopathological diagnosis, apoptosis of the hematolymphoid cells, due to the steroid therapy, may have caused a reduction in the number of large B-cells. A similar effect on the background T-cells and histiocytes, might account for an increased difficulty in assuring a diagnosis $[6,8]$.

Glucocorticoid treatment may have affected the patients in additional manners. Patient 2 shows micronodules that are sclerotic in part. This patient, from the Emory University Hospital, was alive with lymphoma 3 months after surgery [3]. In cases 1 and 2 some epithelioid granulomas were found in the white pulp [3]. The 3 Israeli cases reached complete remission, lasting 12-36 months from diagnosis [6-8]. These findings contrast with Dogan's et al. [1] series, in which 7 of the 12 patients with follow-up, had died of the tumor within 2 years.

The most recent case of MTLBL, diagnosed in our medical center, features a 65-year-old woman (L.Y.) living in a kibbutz (collective community) and employed at a faucet manufacturing plant. She had presented with recent weight loss, night sweats, and pancytopenia. Following an unremarkable bone marrow biopsy, a tru-cut splenic biopsy did not contribute any further to the diagnosis. Splenectomy revealed a spleen of $470 \mathrm{~g}$ with a nodular surface. The cut section displayed fine nodularity, entailing mainly the white pulp.

Immunophenotyping confirmed the picture displayed in our 3 prior cases: of note numerous small T-cells were found, without pan-T-cell restriction and with polyclonal TCRg and TCRb; and an excess of nonepithelioid histiocytes (shown in Fig. 1). A limited population of large Bcells displayed the immunophenotype: CD79a+; CD20-; BCL-2+; BCL-6+, and CD30+ (shown in Fig. 2). We are not aware of the cause of the CD20-negative expression in this patient's spleen although we have documented that the large lymphoid cells are indeed large B cells. Moreover, a PCR analysis demonstrated a monoclonal immunoglobulin heavy chain rearrangement.

A list of medications administered to this patient disclosed a prednisone treatment of $100 \mathrm{mg} /$ day for 4 days. This "pulse" therapy was administered 8 months prior to splenectomy. The prescription was intended for a patient highly suspected for an aggressive lymphoma. Four 

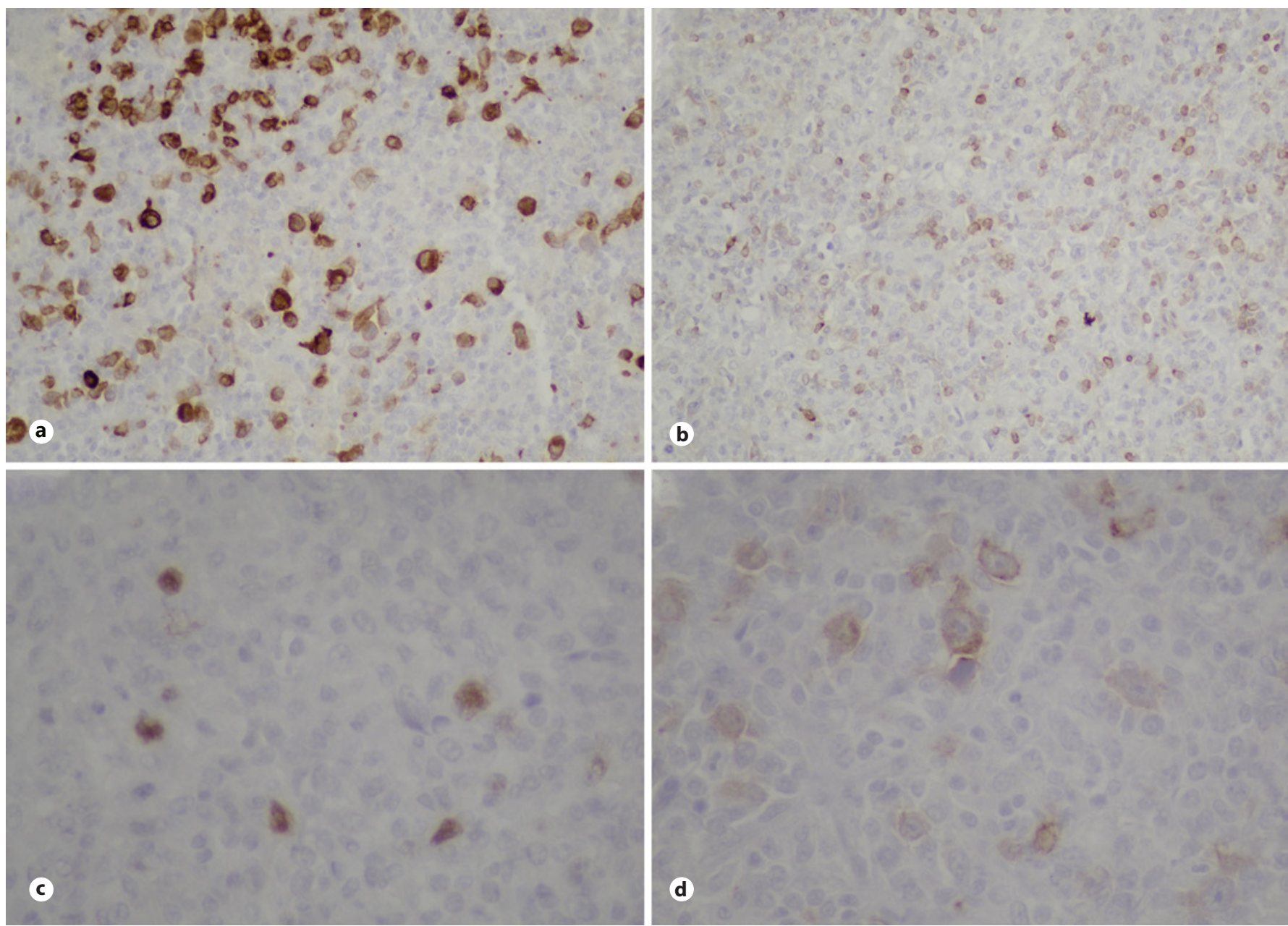

Fig. 2. Immunophenotype of the large lymphoid cells within the splenic micronodules. a A pan-B-cell marker (CD79a) is positive. $\mathbf{b}$ BCL-2 is expressed in the large lymphoid cells. c BCL-6 is expressed in scattered large lymphoid cells. $\mathbf{d}$ CD30 is positive in the large cells.

months after diagnosis the patient is still undergoing RCHOP therapy.

The Putative Role of Steroids Prescribed prior to the

\section{Diagnosis of Lymphoma}

Various cells are affected differently by corticosteroids, by modification of the cellular metabolism or of gene expression. Lymphocytes are among the most affected [9]; these include thymocytes [10], T-lymphocytes [11], B-lymphocytes [12], monocytes, and macrophages [13]. These cells are apparently reduced in number and/ or in size.

Corticosteroids have also an immunosuppressive effect, which is noted in association with lymphomas. Primary central nervous system DLBCL seems to be particu- larly prone to steroid treatment [14]; post-treatment: high rates of nondiagnostic biopsies have been reported, and in several of the diagnostic biopsies, there was extensive apoptosis with only few scattered large neoplastic cells identified on the background of abundant histiocytes. Nevertheless, a mechanism is yet to be uncovered, suggesting a direct influence of steroids on lymphomas. Of note, corticoids have shown a capacity to initiate, as a single agent, the tumor lysis syndrome in aggressive lymphomas $[15,16]$. The morphological changes seen in other B-cell lymphomas with corticosteroid pretreatment has been discussed previously [6]; however, the current authors have not been able to identify a paper relating to corticoid pretreatment in nodal/extranodal involvement by THRLBCL. 
Table 1. Splenic MTLBL - Israeli patients

\begin{tabular}{|c|c|c|c|c|c|}
\hline & \multicolumn{5}{|l|}{ Patient } \\
\hline Gender & $\mathrm{F}$ & M & $\mathrm{F}$ & $\mathrm{F}$ & $\mathrm{F}$ \\
\hline Symptoms & Weight loss & B-symptoms & B sym, pruritus & B sym, cytopenia & Anemia \\
\hline Signs & Splenomegaly & Splenomegaly & Splenomegaly & Splenomegaly & Splenomegaly \\
\hline Dosage & $60 \mathrm{mg} / \mathrm{d}$ & $60 \mathrm{mg} / \mathrm{d}$ & $40 \mathrm{mg} / \mathrm{d}$ & $100 \mathrm{mg} / \mathrm{d} \times 4$ & Unknown \\
\hline Duration & $6 \mathrm{mo}$ & Several months & $3 \mathrm{mo}$ & $4 d$ & $3 \mathrm{mo}$ \\
\hline Indication & Hemol anemia & FUO & Dermatitis & Susp. Lymphoma & Susp. sarcoid \\
\hline Med. history & Asthma & OB. DM, smoke & OB. DM. HB & DM & Granulomatous inflammation \\
\hline Preliminary Dg & $\mathrm{NHL}$ & $\mathrm{NHL}$ & $\mathrm{NHL}$ & $\mathrm{NHL}$ & Sarcoidosis \\
\hline Therapy & $\mathrm{R}-\mathrm{CHOP} \times 6$ & $\mathrm{R}-\mathrm{CHOP} \times 6$ & $\mathrm{R}-\mathrm{CHOP} \times 6$ & $\mathrm{R}-\mathrm{CHOP} \times 6$ & Not mentioned \\
\hline
\end{tabular}

FUO, fever of unknown origin; $\mathrm{OB}$, obesity; $\mathrm{DM}$, diabetes mellitus; $\mathrm{NHL}$, non-Hodgkin lymphoma; $\mathrm{CR}$, complete response; $\mathrm{MZBCL}$, marginal zone $\mathrm{B}$-cell lymphoma; MTLBL, micronodular T-cell/histiocyte-rich large B-cell lymphoma.

\section{Clinical Features in the Israeli Patients}

The major role imputed to the corticoid-induced apoptosis in hematolymphoid conditions has been discussed [6]. Of the 31 pretreated patients mentioned by Kan et al. [6], 8 were given corticosteroids for asthma; 2 for COPD; 5 for autoimmune hemolytic anemia; 3 for neurological symptoms; 3 for dermatoses; 2 for lung infiltrates, and another 10 for miscellaneous conditions (also see Table 1). The corticosteroid dosage ranged from $2 \mathrm{mg} /$ day for 10 years to $100 \mathrm{mg} /$ day for 4 days. Of note, 2 patients were treated with budesonide inhalation; 2 received dexamethasone for 1-2 days by injection.

The question of corticosteroid over-prescription was brought up to a highly experienced hematologist and coauthor (I.L.). He agreed as to the tendency of internists and hematologists, at least in our area, to widely distribute corticosteroids to their patients, given a minimal indication. The low threshold for prescribing corticosteroids may cause an under-reporting of their use in medical communications, namely in pathology requisition forms, as seen for other widely used medications whose implication in histopathologic interpretation is minimal (e.g., acetaminophen and antihistamines). For the reasons presented in this manuscript, we are thoroughly opposed to the lack of transparency in steroid use on requisition forms, as well as caution against their overuse, given the possibility for ultimately creating diagnostic challenges.

An indication for corticosteroids premedication should probably be considered, in cases suspected of non-
Hodgkin lymphoma, displaying B-symptoms, splenomegaly, and/or hypersplenism. This should be contemplated, especially if the preparatory phase for splenectomy is meant to be extended.

\section{Comparison of MTLBL with DLBCL-NOS, \\ THRLBCL, and NLPHL \\ MTLBL}

Stands out for the most marked splenomegaly, the most prominent B-symptoms, severe hypersplenism, together with variable cytopenia. Corticosteroid therapy, preceding splenectomy, may reduce the degree of the splenomegaly, the splenectomy complications, and it may significantly improve the outcome.

\section{THRLBCL}

It affects predominantly males (1.7:1). It represents $10 \%$ of DLBCL-NOS. It shows a predilection for lymph nodes, but may affect the spleen as well, and, with rare exceptions will show a splenic morphology which is either multifocal or micronodular, as with MTLBL. The clinical picture at presentation includes high fever, splenomegaly, and advanced stages. Until recently, the prognosis was believed to be poor. However, this might not be the case anymore.

\section{DLBCL-NOS}

Half the cases emerge in stage I-II. The spleen is frequently enlarged, uni-to multicentric in structure, and the disease 
Table 2. Differential diagnoses of MTLBL and their clinical features

\begin{tabular}{|c|c|c|c|c|}
\hline & \multicolumn{4}{|l|}{ Entity } \\
\hline Lymph node regions & Splenic hilus & Variable & Superficial, mesenteric & Superficial, deep \\
\hline B-signs & Often & May be & None & Fever \\
\hline Other clinical features & Variable cytopenia & $\begin{array}{l}50 \% \text { stages } \\
|-| \mid\end{array}$ & $\begin{array}{l}10 \% \text { stages } \\
\text { III-IV }\end{array}$ & Lymphadenopathy \\
\hline Histology (large B-cells) & $\begin{array}{l}\text { Centroblastic } \\
\text { HRS-like cells }\end{array}$ & $\begin{array}{l}\text { Centroblastic } \\
\text { Immunoblastic } \\
\text { Anaplastic }\end{array}$ & $\begin{array}{l}\text { LP cells } \\
\text { HRS-like cells }\end{array}$ & $\begin{array}{l}\text { Centroblastic } \\
\text { HRS-like cells }\end{array}$ \\
\hline
\end{tabular}

LP cells, lymphocyte predominant (popcorn) cells; HRS-like cells, Hodgkin-Reed-Sternberg-like cells; GC B-cell, Germinal Center B-cell; NGS, nextgeneration sequencing; MTLBL, micronodular T-cell/histiocyte-rich large B-cell lymphoma; DLBCL, diffuse large B-cell lymphoma; THRLBCL, T-cell/histiocyterich large B-cell lymphoma; NLPHL, nodular lymphocyte predominant Hodgkin lymphoma.

progresses rapidly. B-symptoms may occur. The 5-year survival is about $60 \%$, pending the patient's age and stage.

\section{NLPHL}

No B-symptoms are present as a rule. The staging reaches III-IV in $10 \%$ of cases only, and splenomegaly occurs subsequently in this subset of patients. No splenectomy is carried out, as a rule, and the prognosis is overall favorable (also see Table 2).

\section{Discussion}

The taxonomy and histogenesis of splenic MTLBL remain unclear, in part because this subset is extremely rare and considered a variant of THRLBCL which by itself, is somewhat controversially considered a type of DLBCLNOS according to the current (4th Revised, 2017) edition of the WHO Classification of Haematopoietic and Lymphoid Tumours, and comprising by itself about $10 \%$ of all DLBCLs [2]. While earlier studies indicated that THRLBCL was a distinct clinicopathological entity with a more aggressive course than DLBCL $[17,18]$, it was later revealed not to be the case and thought that THRLBCL was just a histologic variant of DLBCL [18]. Later studies still found greater similarity between this entity and NLPHL, both in terms of histology and (to various degrees) immunophenotype [18], as well as of the epidemiology and clinical outcomes [19].

Corticosteroid Pretreatment in Splenic MTLBL
Although THRLBCL arises usually de novo, in some cases this lymphoma results from the progression of NLPHL. Of note, these 2 subsets are not easily segregated [2]. However, this issue has been dealt with extensively elsewhere.

A recent case report on a patient from Syria [20] even presents a "composite" lymphoma elaborated by THRLBL combined with NLPHL. It is significant that the spleen is involved much less frequently in NLPHL and that, only in advanced stages [21]. Higher stages at presentation are rare $[22,23]$. In one report, only 4 of 33 patients with lymph node-based NLPHL exhibited splenomegaly at diagnosis [24]. Along those lines, just as nodal THRLBCL was initially thought to be more aggressive than DLBCL, the prognosis may be eventually more favorable.

Splenic MTLBL, considered by many as associated with the mainly nodal THRLBCL, is a rare variant large B-cell lymphoma $[25,26]$. However, THRLBCL mainly affects lymph nodes, with only limited involvement of bone marrow, liver, lungs, and spleen. In MTLBL, splenomegaly is the predominant trait, accompanied by hypersplenism and B-symptoms. When present, involved lymph nodes are restricted to the splenic hilar region. Most of the cases described present with splenomegaly of variable degree, hypersplenism and cytopenia, and fewer numbers have fever. In only a few patients the disease involves sites beyond the spleen, bone marrow, lungs and splenic hilar lymph nodes. Why do the changes concern 
the spleen, liver, bone marrow, lungs and selectively the splenic hilar lymph nodes, and are not found, as a rule, in peripheral or deep lymph nodes, which are involved preferentially in the THRLBCL?

The presenting symptoms, mainly hypersplenism, when associated with a relatively long period of observation and of preparation for the splenectomy, have evoked the necessity for steroid premedication. In THRLBCL, the response to chemotherapy is poor. Although prednisone is often part of the treatment protocol, a distinct response to corticosteroids as seen punctually in our review has not been assessed.

Regarding 2 Israeli MTLBL patients, the reviewers overlooked completely or temporarily, the corticosteroid pretreatment. It might be bold to assume a similar oversight in 17 unrelated patients [1]. On the other hand, we cannot establish unequivocally that Dogan's patients did not receive pre-splenectomy corticosteroids. While the differences in the severity of splenomegaly must still be underlined in Dogan's cohort, we must agree with these authors that most if not all, did not receive corticoid pretreatment. Special attention should be given to this therapeutic modality in the future, and it should not be taken for granted [1].

\section{Conclusion}

The apparent, complete absence of a corticoid premedication in all the cases of MTLBL from most cases recorded is contrasted with the 4 cases diagnosed in BeerSheva, Israel, and with a single case from Emory University Hospital, all of which were given steroid pretreatment. Glucocorticoids were administered to 3 of the 4 Israeli patients in relation to the ongoing disease: hemolytic anemia, fever of unknown origin, and weight loss. In the fourth patient, the pretreatment was directed at dermatitis. The case reported by $\mathrm{Li}$ et al. [3] was pretreated with corticoids following the suspicion of sarcoidosis.

Evidence is presented of a significant role for corticosteroid premedication prior to splenectomy in MTLBL regarding an apparent transformation from the classic THRLBCL morphology from which the splenic form of the disease seems to derive. Indications for its prescription comprise on the one hand splenomegaly, B-symptoms, hypersplenism, with or without cytopenia. On the other hand, intercurrent conditions, like asthma, COPD, and so on, might serve as a pretext, in case the patient is highly symptomatic, but does not exhibit specific symptoms and signs of the lymphoma. A limitation in our narrative regards the degree of confidence attributed to cor- ticosteroid therapy in MTLBL. This medication, pertinent if the 4 Israeli patients could be classified as stages III or IV of the non-Hodgkin's lymphoma, right prior to the prednisone prescription. However, such a query remains insoluble, as one may never know what the stage might be immediately before splenectomy. The issue is equally pertinent for staging in the remaining MTLBL cases $[26,27]$. Our thesis, surmising that a greater proportion of MTLBL patients had undergone pre-diagnostic corticosteroid treatment than formerly realized, may be admissible, consideration given to 2 Israeli cases in which steroid pretreatment was overlooked at review.

\section{Statement of Ethics}

Written informed consent was obtained from the patient of the most recent Israeli case of MTLBL, being presented in this paper for the first time, for publication of this case report and any accompanying images.

\section{Conflict of Interest Statement}

The authors have no conflicts of interest to declare.

\section{Funding Sources}

No funding was provided for the preparation of the manuscript.

\section{Author Contributions}

The following were the authors' contributions: B.S.: resources, visualization, and writing - review and editing; K.N.: investigation, resources, visualization, and writing - review and editing; D.B.: investigation, methodology, item conception, supervision, and writing original draft; and I.L.: resources and writing - review and editing.

\section{References}

1 Dogan A, Burke JS, Goteri G, Stitson RN, Wotherspoon AC, Isaacson PG. Micronodular T-cell/histiocyte-rich large B-cell lymphoma of the spleen: histology, immunophenotype, and differential diagnosis. Am J Surg Pathol. 2003;27:903-11.

2 Ott G, Delabie J, Gascoyne R, Campo E, Stein $\mathrm{H}$, Jaffe E. T-cell/histocyte-rich large B-cell lymphoma. In: Swerdlow SH, Campo E, Harris NL, Jaffe ES, Pileri SA, Stein H, et al., editors. WHO classification of tumours of haematopoietic and lymphoid tissues. Revised 4. Lyon: IARC; 2017. p. 298-9. 
3 Li S, Mann KP, Holden JT. T-cell-rich B-cell lymphoma presenting in the spleen: a clinicopathologic analysis of 3 cases. Int J Surg Pathol. 2004;12(1):31-7.

4 Dincol G, Agan M, Dogan O, Diz-Kucukkaya R, Uslu B. T-cell-rich B-cell lymphoma of the spleen presenting with severe hypersplenism. Clin Lab Haematol. 2006;28:419-22.

5 Sinit RB, Dorer RK, Flores JP, Aboulafia DM. Rare causes of isolated and progressive splenic lesions: challenges in differential diagnosis, evaluation, and treatment of primary splenic lymphomas. Clin Med Insights Blood Disord. 2020;13:1179545X20926188.

6 Kan E, Levi I, Benharroch D. Alterations in the primary diagnosis of lymphomas pretreated with corticosteroid agents. Leuk Lymphoma. 2011;52:425-8.

7 Kan E, Levy I, Benharroch D. Splenic micronodular T-cell/histiocyte-rich large B-cell lymphoma. Ann Diagn Pathol. 2008;12:2902.

8 Kan E, Levy I, Benharroch D. Splenic micronodular T-cell/histiocyte-rich large B-cell lymphoma: effect of prior corticosteroid therapy. Virchows Arch. 2009;455:337-41.

9 Wood AC, Elvin P, Hickman JA. Induction of apoptosis by anti-cancer drugs with disparate modes of action: kinetics of cell death and changes in c-myc expression. Br J Cancer. 1995;71:937-41.

10 Pruschy M, Shi Y-Q, Crompton NEA, Steinbach J, Aguzzi A, Glanzmann C, et al. The proto-oncogene c-fos mediates apoptosis in murine T-lymphocytes induced by ionizing radiation and dexamethasone. Biochem Biophys Res Commun. 1997;241:519-24.
11 Herold MJ, McPherson KG, Reichardt HM. Glucocorticoids in T cell apoptosis and function. Cell Mol Life Sci. 2006;63:60-72.

12 Andréau K, Lemaire C, Souvannavong V, Adam A. Induction of apoptosis by dexamethasone in the $\mathrm{B}$ cell lineage. Immunopharmacology. 1998;40:67-76.

13 Schmidt M, Pauels HG, Lügering N, Lügering A, Domschke W, Kucharzik T. Glucocorticoids induce apoptosis in human monocytes: potential role of IL-1 beta. J Immunol. 1999; 163:3484-90.

14 Binnahil M, Au K, Lu JQ, Wheatley BM, Sankar T. The influence of corticosteroids on diagnostic accuracy of biopsy for primary central nervous system lymphoma. Can J Neurol Sci. 2016;43:721-5.

15 Sparano J, Ramirez M, Wiernik PH. Increasing recognition of corticosteroid-induced tumor lysis syndrome in non-Hodgkin's lymphoma. Cancer. 1990;65:1072-3.

16 Lerza R, Botta M, Barsotti B, Schenone E, Mencoboni M, Bogliolo G, et al. Dexamethazone-induced acute tumor lysis syndrome in a T-cell malignant lymphoma. Leuk Lymphoma. 2002;43:1129-32.

17 Achten R, Verhoef G, Vanuytsel L, de WolfPeeters C. T-cell/histiocyte-rich large B-cell lymphoma: a distinct clinicopathologic entity. J Clin Oncol. 2002;20:1269-77.

18 Lim MS, Beaty M, Sorbara L, Cheng RZ, Pittaluga S, Raffeld M, et al. T-cell/histiocyterich large B-cell lymphoma. Am J Surg Pathol. 2002;26:1458-66.
19 Ollila TA, Reagan JL, Olszewski AJ. Clinical features and survival of patients with T-cell/ histiocyte-rich large B-cell lymphoma: analysis of the National Cancer Data Base. Leuk Lymphoma. 2019;60:3426-33.

20 Esper A, Alhoulaiby S, Zuhri Yafi R, Alshehabi Z. Composite lymphoma of T-cell rich, histiocyte-rich diffuse large B-cell lymphoma and nodular lymphocyte predominant Hodgkin lymphoma: a case report. J Med Case Rep. 2021;15(1):163. Undefined.

21 Stein H, Swerdlow SH, Gascoyne RD, Popemma S, Jaffe ES, Pileri SA. Nodular lymphocyte predominant Hodgkin lymphoma. WHO Classification of Tumours of Haematopoietic and Lymphoid Tissues, Revised 4. Lyon: IARC; 2017. p. 431-4.

22 Ozari A, Arber DA. Spleen: normal architecture and neoplastic and non-neoplastic lesions. In: Jaffe ES, Harris NL, Arber DA, editors. Hematopathology. 2nd ed. Philadelphia: Elsevier; 2017. p. 1118-9.

23 Kraus MD, Fleming MD, Vonderheide RH. The spleen as a diagnostic specimen: a review of 10 years' experience at two tertiary care institutions. Cancer. 2001;91:2001-9.

24 Mollejo M, Algara P, Mateo MS, Menárguez J, Pascual E, Fresno MF, et al. Large B-cell lymphoma presenting in the spleen. Am J Surg Pathol. 2003;27:895-902.

25 Fan L, He F, Huang G. Micronodular T-cell/ histiocyte-rich B-cell lymphoma in spleen. $\mathrm{Br}$ J Haematol. 2009;146:126. Undefined.

26 Pittaluga S, Jaffe ES. T-cell/histiocyte-rich large B-cell lymphoma. Haematologica. 2010; 95:352-6.

27 Betman HF, Vardiman JW, Lau J. T-cell-rich B-cell lymphoma of the spleen. Am J Surg Pathol. 1994;18:323-4. 